\title{
TWO INDEX LAWS FOR FRACTIONAL INTEGRALS AND DERIVATIVES
}

\author{
E. R. LOVE \\ (Received 27 February 1970) \\ Communicated by B. Mond
}

\begin{abstract}
SUMMARY
The first index law, or addition theorem, is well known. The second is much less well known; but both have been found to be of importance in recent studies of hypergeometric integral equations. The first law has usually been considered only in the simple case of orders of integration which have positive real part, or in the context of generalized functions. Arising out of the need to manipulate expressions involving several fractional integrals and derivatives, our aim here is to establish both laws for all combinations of complex orders of integration and differentiation, and for nearly all functions for which the fractional derivatives involved exist as locally integrable functions.
\end{abstract}

\section{Introduction}

The bare formal statements of the laws in terms of the fractional integral operator $I^{c}$ (for definitions see $\$ 3$, in particular (5) to (8)) are:

$$
I^{b} I^{a} f=I^{a+b} f,
$$

where $a$ and $b$ are any complex numbers, and

$$
I^{c} x^{b} I^{a} f(x)=x^{-a} I^{-b} x^{-c} f(x),
$$

where also $a+b+c=0$.

Both have been known for a long time, (2) less well than (1), in the cases where the orders of integration (indices of $I$ ) are positive or have positive real parts. But cases involving fractional derivatives (orders of integration having real part negative or zero) have been needed, as in [11], and continue to be needed in further work on hypergeometric integral equations. This paper has arisen out of that need, and it is hoped that it will serve as a comprehensive reference, giving conditions under which (1) and (2) hold for locally integrable functions and for all possible sign combinations of $\alpha, \beta, \gamma$ (the real parts of $a, b, c$ ).

An instance of (2) in which $a$ and $c$ are not both positive can be seen in 
Widder [1, p. 17, Lem. 3.11]. If $k$ is a positive integer, $D$ is the familiar differentiation operator and $D^{2 k-1} f$ exists, then

$$
D^{k} x^{2 k-1} D^{k-1} f(x)=x^{k-1} D^{2 k-1} x^{k} f(x) .
$$

Widder proves this by applications of Leibniz's rule for differentiation of products. Its connection with (2) is immediately apparent if we replace $D$ by $I^{-1}$. Some less special instances of (2) will be mentioned in $\$ 2$.

In order to avoid what appear to be artificial exceptions when one or more of the indices $a, b, c$ is purely imaginary, fractional derivatives (or integrals) of purely imaginary orders are needed. For this purpose reference is made to [12], and the definitions and other features of that paper needed here are summarized in $\$ 3$, together with some other preliminaries of a fairly novel character.

There is perhaps some interest in re-writing (1) and (2) in the following way, using formal inversions:

$$
\begin{aligned}
I^{c} I^{b} I^{a} f=f & \text { where } a+b+c=0, \\
x^{c} I^{b} x^{a} I^{c} x^{b} I^{a} f=f & \text { where } a+b+c=0 .
\end{aligned}
$$

However (1) and (2) are the forms which have arisen so far.

\section{Related Investigations}

After the earliest mentionings, [2], [3], [4] and others, of the first law (1) for complex $a$ and $b$ with positive real parts, Kober [5] defined $I^{c} f$ for re $c=0$ using Mellin transforms. He proved (1) when re $a=0=$ re $b$, for functions $f$ in a certain subspace of $L^{p}(0, \alpha)$ when $p \geqq 2$. Riesz [6] gave a definition of $I^{c} f$ for re $c \leqq 0$, but his proof of (1) assumes (p. 12) that $f$ has $p$ continuous derivatives, where $p>\max (-\operatorname{re} a,-\operatorname{re}(a+b))$. Gelfond and Shilov [8] define $I^{c} f$ as a generalized function, and derive (1) without restriction on $a$ and $b$; but this treatment lacks conditions under which the fractional derivatives occurring are identifiable with ordinary functions. Erdélyi [10] mentions a definition of $I^{c} f$ when re $c>-p$ using finite part integrals, but treats it by the methods of [8] rather than independently.

In $\S 4$ of this paper the first law (1) is proved for all complex $a$ and $b$, assuming only that the fractional derivatives occurring in it exist as locally integrable functions. This is not at all a difficult matter, but it is a necessary preliminary to the main task of this paper, which is to prove similar results for the second law (2).

The second law (2) was proved, for re $a>0$ and re $c>0$, by Kober [4, p. 201], actually in a disguised form. Translating into our notation two of his four equations (3.7), namely

$$
I_{\eta, \lambda}^{+}\left(I_{\eta+\lambda, \mu}^{+} f\right)=I_{\eta, \lambda+\mu}^{+} f=I_{\eta+\lambda, \mu}^{+}\left(I_{\eta, \lambda}^{+} f\right) .
$$


we obtain, using his definition (1.1) and our definition (5), and putting $g(x)=x^{\eta+\lambda} f(x)$

$$
I^{\lambda} x^{-\lambda-\mu} I^{\mu} g(x)=x^{-\mu} I^{\lambda+\mu} x^{-\lambda} g(x)=x^{-\mu} I^{\mu} I^{\lambda} x^{-\lambda} g(x)
$$

for re $\lambda>0$, re $\mu>0$ and rather restricted $g$. These are essentially our (2) and (1), with orders of integration having positive real parts.

The second law (2) underlies a paper by Higgins [9] which shows in a formal way its connection with many important formulae. His equation (17) on p. 8 is equivalent to our (2) by applying the operator $I_{t}^{-\alpha-\beta-\gamma}$. The same law (2) appears in $[11$, p. 175, Thm. 2] where, replacing $\lambda, a, b$ and $f(t)$ by $c, c+a, a$ and $t^{-c-a} f(t)$ respectively, we obtain

$$
I^{c} x^{-c-a} I^{a} f(x)={ }^{0} x^{-a} I^{a+c} x^{-c} f(x)
$$

for suitable $f$ when re $a>0$ and re $c>0$.

In $\S \S 5,6,7$ of this paper the second law (2) is proved for all complex $a, b, c$ st:ch that $a+b+c=0$. This involves extended treatment of thirteen different sign combinations, under conditions which require at most a little more than bare existence of the fractional derivatives involved.

\section{Definitions and Preliminaries}

The main sources of these are [11] and [12].

All functions considered are complex-valued measurable functions defined almost everywhere on the real half-line $(0, \infty)$. Two functions are regarded as equal if they are equivalent, that is, have equal values almost everywhere. Local integrability always means local integrability in $[0, \infty)$; consequently it means $\mathrm{n}$ tegrable on $[0, l]$ for each $l>0$.

Fractional integrals. If re $c>0$ and $f$ is a function, $I^{c} f$ is defined as the function (if any) whose values are

$$
{ }^{R} f(x)=\int_{0}^{x} \frac{(x-t)^{c-1}}{\Gamma(c)} f(t) d t .
$$

THEOREM A. If re $c>0$ and $f$ is a locally integrable function, then $I^{c} f$ is a locally integrable function.

THEOREM B. If re $a>0$, re $b>0$ and $f$ is a locally integrable function, then

$$
I^{b}\left(I^{a} f\right)=I^{a+b} f .
$$

Fractional derivatives. If $\mathrm{re}(-c)<0$ and $f$ is a function, $I^{-c} f$ is defined as a locally integrable function $\phi$ (if any) such that

$$
I^{c} \phi=f,
$$


where $I^{c} \phi$ is defined as in (5). It is well known that $I^{-c} f$, if it exists at all, is a single class of equivalent functions.

Derivatives of imaginary order. If re $c=0$ and $f$ is a function, $I^{c} f$ is defined by

$$
I^{c} f=I^{-1} I^{1+c} f
$$

if the right side exists in accordance with (5) and (6).

LEMMA C. If re $c=0$ and $f$ is locally integrable, then $I^{1+c} f(x)$ is defined for all $x \geqq 0$, is continuous in $x \geqq 0$ and vanishes at $x=0$.

This is Lem. 1 of [12].

THEOREM D. If re $c=0$ and $f$ is locally integrable, then $I^{c} f$ exists if and only if $I^{1+c} f$ is locally absolutely continuous. In that event $I^{c} f$ is equivalent to its almost everywhere derivative.

This is Thm. 1 of [12].

THEOREM E. If $f$ is locally integrable and $c$ is any complex number such that $I^{c} f$ exists, then $I^{c} f$ is locally integrable. Also $I^{-c} I^{c} f$ exists and is equivalent to $f$.

This is a combined statement of Thm. 1 Cor. and Thm. 2 Cor. of [12].

Some elementary integrals. It is convenient to prove Lemmas F, G, H at this stage.

LEMMA F. If $\lambda>0$ and $0<t<x$, then

$$
\int_{t}^{x}(s-t)^{\lambda-1} s^{\mu} d s= \begin{cases}O\left(x^{\lambda+\mu}\right) & \text { if } \lambda+\mu>0, \\ O\left(t^{\lambda+\mu}\right) & \text { if } \lambda+\mu<0,\end{cases}
$$

the bounds implied in both $O$-symbols being independent of $x$ and $t$.

If $\mu>0$, the integral is less than

$$
\int_{t}^{x}(s-t)^{\lambda-1} x^{\mu} d s=\frac{(x-t)^{\lambda}}{\lambda} x^{\mu}<\frac{x^{\lambda+\mu}}{\lambda} .
$$

If $0 \geqq \mu>-\lambda$, the integral is at most

$$
\int_{t}^{x}(s-t)^{\lambda+\mu-1} d s=\frac{(x-t)^{\lambda+\mu}}{\lambda+\mu}<\frac{x^{\lambda+\mu}}{\lambda+\mu} .
$$

If $\lambda+\mu<0$, the integral is less than

$$
\int_{t}^{\infty}(s-t)^{\lambda-1} s^{\mu} d s=t^{\lambda+\mu} \int_{1}^{\infty}(v-1)^{\lambda-1} v^{\mu} d v
$$

putting $s=t v$; and the last integral is convergent.

Lemma G. If $a+b+c=0$, re $a>0$, re $b<0$, re $c>0$ and $0<t<x$, then 


$$
\int_{t}^{x} \frac{(x-s)^{c-1}}{\Gamma(c)} s^{b} \frac{(s-t)^{a-1}}{\Gamma(a)} d s=x^{-a} \frac{(x-t)^{-b-1}}{\Gamma(-b)} t^{-c} .
$$

The integral is absolutely convergent, and the substitution $s=1 / u$ transforms it into

$$
\frac{x^{c-1}}{\Gamma(c)} \frac{t^{a-1}}{\Gamma(a)} \int_{1 / x}^{1 / t}\left(u-\frac{1}{x}\right)^{c-1}\left(\frac{1}{t}-u\right)^{a-1} d u=\frac{x^{c-1} t^{a-1}}{\Gamma(c+a)}\left(\frac{1}{t}-\frac{1}{x}\right)^{c+a-1}
$$

Lemma H. If $a+b+c=0$, re $a>0$, re $b<0$, re $c>-1$ and $0<t<x$, then

$$
\int_{t}^{x} \frac{(x-s)^{c}}{\Gamma(c+1)} s^{b} \frac{(s-t)^{a-1}}{\Gamma(a)} d s=\int_{t}^{x} s^{-a} \frac{(s-t)^{-b-1}}{\Gamma(-b)} t^{-c} d s .
$$

The substitution $s=t+(x-t) u$ converts the left side into

$$
\begin{aligned}
(x-t)^{c+a} t^{b} \int_{0}^{1} \frac{(1-u)^{c}}{\Gamma(c+1)} \frac{u^{a-1}}{\Gamma(a)}\left(1+\frac{x-t}{t} u\right)^{b} d u \\
\quad=\frac{(x-t)^{c+a}}{\Gamma(c+a+1)} t^{b} F\left(-b, a, c+a+1,1-\frac{x}{t}\right)
\end{aligned}
$$

using Euler's integral for the hypergeometric function $F[7,2.1(10)]$.

The right side of the proposed equation can be written as an integral of the same form as the left, and thence expressed in terms of the hypergeometric function using the last equation. The right side is, in fact,

$$
t^{-c} \int_{t}^{x} \frac{(x-s)^{0}}{\Gamma(1)} s^{-a} \frac{(s-t)^{-b-1}}{\Gamma(-b)} d s=\frac{(x-t)^{-b}}{\Gamma(1-b)} t^{-c-a} F\left(a,-b, 1-b, 1-\frac{x}{t}\right) .
$$

The equality of the two sides when $c+a=-b$ now follows from the symmetry of the hypergeometric function in its first two parameters.*

Composite operations. $I^{c} x^{b} I^{a} f(x)$ means the function got from $f(x)$ by forming successively $I^{a} f(x), x^{b} I^{a} f(x)$ and $I^{c}\left\{x^{b} I^{a} f(x)\right\}$; this notation is consistent with the customary understanding of the left side of (3). In particular, if re $a>0$ and $\operatorname{re} c>0$, the value of this function at $x$ is

$$
I^{c} x^{b} I^{a} f(x)=\int_{0}^{x} \frac{(x-s)^{c-1}}{\Gamma(c)} s^{b} d s \int_{0}^{s} \frac{(s-t)^{a-1}}{\Gamma(a)} f(t) d t .
$$

An integrability class. $Q_{q}$ is defined as the class of functions $f$ such that $x^{q} f(x)$ is locally integrable in $[0, \infty)$ (the case $d=\infty$ of 11, p. $180,(5.4)$ ). In particular, $Q_{0}$ consists of all locally integrable functions.

Clearly $Q_{q}$ expands as the real parameter $q$ increases. As we consider mainly locally integrable functions the subscript $q$ will usually be negative.

* An alternative proof can be given by integrating the equation of Lemma $G$ with respect to $x$ and inverting the order of integration on the left. However it is then necessary, first to restrict $c$ to re $c>0$, and then to justify analytical continuation of both sides into re $c>-1$. 
The next theorem, or rather the case $q<$ re $a$ of it, is related to a theorem of Kober [4, p. 199, Thm. 2(3.4)(i)]; it is also discussed in [11, p. 174, Remark 4]. In both places it is a corollary of a much more elaborate theorem, and it seems appropriate to give here an independent simple proof that covers the other cases as well.

THEOREM K. If $a$ is any complex number, re $c>0$ and $f \in Q_{q}$, then

$$
x^{-a-c} I^{c} x^{a} f(x) \begin{cases}\text { is in } Q_{q} & \text { if } q<\mathrm{re} a, \\ \text { is in } Q_{q+\varepsilon} \text { for each } \varepsilon>0 & \text { if } q=\mathrm{re} a, \\ \text { may not exist } & \text { if } q>\operatorname{re} a .\end{cases}
$$

For the third case $f(x)=x^{-a-1}$ is in $Q_{q}$ but $I^{c} x^{-1}$ does not exist.

For the first two cases $x^{a} f(x)=x^{a-q} x^{q} f(x)$ is locally integrable, so $I^{c} x^{a} f(x)$ exists and is locally integrable by Theorem A. So the function in question is measurable. Write $\alpha=$ re $a, \gamma=\operatorname{re} c>0$, and suppose that $r \geqq q$. Then, for each $l>0$,

$$
\begin{aligned}
& |\Gamma(c)| \int_{0}^{l}\left|x^{r-a-c} I^{c} x^{a} f(x)\right| d x=\int_{0}^{l} x^{r-\alpha-\gamma}\left|\int_{0}^{x}(x-t)^{c-1} t^{a} f(t) d t\right| d x \\
& \leqq \int_{0}^{l} x^{r-\alpha-\gamma} d x \int_{0}^{x}(x-t)^{\gamma-1} t^{\alpha}|f(t)| d t=\int_{0}^{l} t^{\alpha}|f(t)| d t \int_{t}^{l}(x-t)^{\gamma-1} x^{r-\alpha-\gamma} d x \\
& \text { (9) }=\int_{0}^{l} t^{r}|f(t)| d t \int_{0}^{l / t-1} \frac{s^{\gamma-1}}{(1+s)^{\gamma+\alpha-r}} d s \text {. }
\end{aligned}
$$

putting $x=t(1+s)$.

If $q<\alpha$ we take $r=q$; then (9) is less than

as required.

$$
\int_{0}^{l} t^{q}|f(t)| d t \int_{0}^{\infty} \frac{s^{\gamma-1}}{(1+s)^{\gamma+\alpha-q}} d s<\infty,
$$

If $q=\alpha$ we take $r=q+\varepsilon$, where $0<\varepsilon<\gamma$; then (9) is less than

as required.

$$
\begin{aligned}
& \int_{0}^{l} t^{q+\varepsilon}|f(t)| d t \int_{0}^{l / t} \frac{s^{\gamma-1}}{(1+s)^{\gamma-\varepsilon}} d s \\
& \quad \leqq \int_{0}^{l} t^{q+\varepsilon}|f(t)| d t\left(\int_{0}^{1} s^{\gamma-1} d s+\int_{1}^{l / t} s^{\varepsilon-1} d s\right) \\
& \quad \leqq \int_{0}^{l} t^{q+\varepsilon}|f(t)| d t\left(\frac{1}{\gamma}+\frac{(l / t)^{\varepsilon}}{\varepsilon}\right) \\
& \quad \leqq\left(\frac{1}{\gamma}+\frac{1}{\varepsilon}\right) l^{\varepsilon} \int_{0}^{l} t^{q}|f(t)| d t<\infty,
\end{aligned}
$$


Counter example. The need for the extra $\varepsilon$ in Theorem $\mathrm{K}$ when $q=$ re $a$ is made evident by the example $q=c=a=1$,

$$
f(x)=\{x \log (2 / x)\}^{-2} \text { if } 0<x<1, f(x)=0 \text { otherwise. }
$$

For $f \in Q_{1}$ since $x f(x)$ is locally integrable; but $x^{-2} I^{1} x f(x) \notin Q_{1}$ because, if $0<x<1$,

$$
x \cdot x^{-2} I^{1} x f(x)=\frac{1}{x} \int_{0}^{x} \frac{1}{t(\log (2 / t))^{2}} d t=\frac{1}{x \log (2 / x)},
$$

and this function is not integrable on $(0,1)$.

Remark. The two remaining preliminary theorems are of a similar nature to Theorem $\mathrm{K}$, but they deal with fractional derivatives rather than integrals, and are consequently more awkward. They are not needed in this paper until Theorem 15 is reached. A proof of Theorem $\mathbf{M}$ is given immediately below, even though it uses Theorem 11; it seems appropriate to locate it here, with the allied Theorems $\mathrm{K}$ and $\mathrm{L}$.

THEOREM L. If $q<0<\mathrm{re} b$ and $q<\mathrm{re} a$, then $x^{-a} I^{-b} x^{a} g(x)$ exists in $Q_{q}$ if and only $I^{-b} g(x)$ also exists in $Q_{q}$.

This is 11, p. 193, Theorem 5 Cor. The main part of the proof is on p. 181-2, and the rest on p. 193.

THEOREM M. If $q<$ re $a<\mathrm{re} b=0, x^{a} g(x)$ is locally integrable and $x^{-a} I^{-b} x^{a} g(x)$ exists in $Q_{q}$, then $I^{-b} g(x)$ also exists in $Q_{q}$.

To prove this, let $f(x)=x^{-a} I^{-b} x^{a} g(x)$. By Theorem $\mathrm{E}$ with $c$ and $f(x)$ replaced by $-b$ and $x^{a} g(x), I^{b} x^{a} f(x)$ exists and is equivalent to $x^{a} g(x)$. So, by (7),

$$
I^{-1} I^{1+b} x^{a} f(x)={ }^{0} x^{a} g(x) .
$$

By (6),

$$
I^{1+b} x^{a} f(x)={ }^{0} I^{1} x^{a} g(x)
$$

and again by (6),

$$
x^{a} f(x)={ }^{0} I^{-1-b} I^{1} x^{a} g(x) ;
$$

the latter because $x^{a} f(x)=x^{a-q} x^{q} f(x)$ is locally integrable, since $f \in Q_{q}$ and $x^{a-q}$ is locally bounded. [Notice that local integrability is an essential part of our definition (6) of a fractional derivative.]

Defining $h(x)=x^{-a} I^{1} x^{a} g(x),(10)$ can be written in the form

$$
f(x)={ }^{0} x^{-a} I^{-1-b} x^{a} h(x) .
$$

Now this right side exists in $Q_{q}$; so Theorem $\mathrm{L}$ with $b$ and $g$ replaced by $1+b$ and $h$ shows that $I^{-1-b} h(x)$ also exists in $Q_{q}$. Defining $\phi=I^{-1-b} h$, we now have that $h=I^{1+b} \phi$ by (6), that $\phi \in Q_{q}$ and that 


$$
x^{a} g(x)={ }^{0} I^{-1} x^{a} h(x)=I^{-1} x^{a} I^{1+b} \phi(x) .
$$

The equations of the preceding paragraph also give

$$
I^{1+b} \phi(x)={ }^{0} x^{-a} \int_{0}^{x} t^{a} g(t) d t .
$$

Since $\phi$ is locally integrable by its definition, $I^{1+b} \phi(x)$ is continuous in $x \geqq 0$ by Lemma $C$. So is the right side of (12) since re $(-a)>0$. So (12) holds for all $x$, not only for almost all.

The right side of (12) is locally absolutely continuous, being the product of two such functions; thus $I^{1+b} \phi$ is locally absolutely continuous. Theorem D now gives that $I^{b} \phi$ exists and is the almost everywhere derivative of $I^{1+b} \phi$. So, performing the differentiation in (11),

$$
x^{a} g(x)={ }^{0} x^{a}\left(\frac{a}{x} I^{1+b} \phi(x)+I^{b} \phi(x)\right) .
$$

Since $\phi \in Q_{q}$, Theorem 11 (see $\S 6$ ) with $a, b, c, \eta$ and $f$ replaced by $1+b$, $-1,-b,-q$ and $\phi$, gives that the function

$$
\psi(x)=I^{-b} x^{-1} I^{1+b} \phi(x)
$$

exists in $Q_{q}$. By Theorem $\mathrm{E}$, with $c$ and $f(x)$ replaced by $-b$ and $x^{-1} I^{1+b} \phi(x)$, $I^{b} \psi(x)$ exists and is equivalent to $x^{-1} I^{1+b} \phi(x)$, provided that the latter is locally integrable, a fact which we now verify.

By (13),

$$
a x^{-1} I^{1+b} \phi(x)={ }^{0} g(x)-I^{b} \phi(x) .
$$

Now $x^{a} g(x)$ is locally integrable, and this property is preserved in multiplying by the locally bounded function $x^{-a}$. So $g$ is locally integrable. So also is $I^{b} \phi$, by Theorem E. Hence so is (14).

We thus have that $I^{b} \psi(x)={ }^{0} x^{-1} I^{1+b} \phi(x)$, where $\psi \in Q_{q}$. Substituting in (13)

$$
g(x)={ }^{0} I^{b}(a \psi(x)+\phi(x)),
$$

and $a \psi+\phi \in Q_{q}$. In particular, $a \psi+\phi$ is locally integrable; so Theorem 4 Cor. (see $\S 4$ ) gives that $I^{-b} g$ exists and is equivalent to $a \psi+\phi$. Thus $I^{-b} g$ exists in $Q_{q}$, as required.

\section{The First Index Law}

The form most suitable for our purpose is stated comprehensively as follows.

If $a$ and $b$ are any complex numbers, $f$ is locally integrable, and $I^{a} f$ and $I^{a+b} f$ exist (as locally integrable functions), then $I^{b} I^{a} f$ exists and

$$
I^{b} I^{a} f=I^{a+b} f .
$$


Remembering $\$ 3,(15)$ means that the two sides are equivalent.

However, for many combinations of values of $a$ and $b$ the existence of $I^{a} f$ or $I^{a+b} f$ need not be assumed, for it is a consequence of Theorem $A$ and the other hypotheses. Theorems 1 to 4 are the resulting simplified theorems. They do in fact cover all complex values of $a$ and $b$.

THEOREM 1. If re $a>0, \operatorname{re}(a+b)>0$ and $f$ is locally integrable, then

$$
I^{b} I^{a} f=I^{a+b} f .
$$

If $\mathrm{re} b>0$ this reduces to Theorem $\mathbf{B}$.

If $\mathrm{re} b=0$, two applications of Theorem $\mathrm{B}$ give

$$
I^{1+b} I^{a} f=I^{1+b+a} f=I^{1} I^{b+a} f
$$

The left side is continuous by Lemma $C$, since $I^{a} f$ is locally integrable by Theorem A. The right side is locally absolutely continuous, since $I^{b+a} f$ is locally integrable by Theorem A. Thus the two extreme members of (16) are identical, not merely equivalent. So the left member is locally absolutely continuous; whence, by Theorem D, $I^{b} I^{a} f$ exists and is equivalent to $I^{b+a} f$.

If re $b<0$, Theorem $B$ gives

$$
I^{-b} I^{a+b} f=I^{a} f .
$$

Now $I^{a+b} f$ is locally integrable by Theorem $A$; this permits, by definition (6), inversion of (17) giving (15).

THEOREM 2. If re $a \leqq 0$, re $b>0, f$ is locally integrable and $I^{a} f$ exists then

$$
I^{b} I^{a} f=I^{a+b} f
$$

Suppose re $a=0$. By Theorem E, $I^{a} f$ is locally integrable; and by Theorem A, both sides of (15) exist and are locally integrable, since re $b>0$ and $\operatorname{re}(a+b)>0$. By four applications of Theorem B,

$$
I^{1} I^{b} I^{a} f=I^{1+b} I^{a} f=I^{b} I^{1} I^{a} f=I^{b} I^{1+a} f=I^{b+1+a} f=I^{1} I^{b+a} f,
$$

the middle step following from definitions (6) and (7). The extreme members of (18) are not only equivalent but identical, since they are continuous. From this the equivalence (15) follows by differentiation.

Suppose re $a<0$. By (6) there is locally integrable $\phi$ such that

$$
I^{-a} \phi=f \text { and } \phi=I^{a} f .
$$

By Theorem A, $I^{b} \phi$ exists; that is, $I^{b} I^{a} f$ exists. And by Theorem 1 , with $f, a$ and $b$ replaced by $\phi,-a$ and $a+b$,

$$
I^{b} I^{a} f=I^{b} \phi=I^{a+b} I^{-a} \phi=I^{a+b} f .
$$


COROLLARY. If re $a<\mathrm{re} b, f$ is locally integrable and $I^{a} f$ exists, then $I^{b} f$ exists.

No proof is needed if re $a \geqq 0$, for then $I^{b} f$ exists by Theorem A. If re $a<0$, Theorem 2 with $b$ replaced by $b-a$ ensures the existence of $I^{b} f$, even if re $b \leqq 0$, since the right side of (15) becomes $I^{b} f$.

THEOREM 3. If re $(a+b) \leqq 0$, re $b<0, f$ is locally integrable and $I^{a+b} f$ exists, then

$$
I^{b} I^{a} f=I^{a+b} f .
$$

By Theorem 2 with $a$ and $b$ replaced by $a+b$ and $-b$, (17) holds. But $I^{a+b} f$ is locally integrable by Theorem $\mathrm{E}$; this permits, by definition (6), inversion of (17) giving (15).

THEOREM 4. If re $a \leqq 0$, re $b=0, f$ is locally integrable and $I^{a} f$ and $I^{a+b} f$ exist, then

$$
I^{b} I^{a} f=I^{a+b} f .
$$

By Theorem 2 with $b$ replaced by $1+b$,

$$
I^{1+b} I^{a} f=I^{1+b+a} f .
$$

Since $I^{a} f$ is locally integrable by Theorem E, the left side of (20) is continuous by Lemma $\mathrm{C}$.

By Theorem 2 with $a$ and $b$ replaced by $a+b$ and 1 ,

$$
I^{1} I^{a+b} f=I^{1+a+b} f .
$$

Again by Theorem E $I^{a+b} f$ is locally integrable, and so the left side of (21) is locally absolutely continuous. Putting (20) and (21) together,

$$
I^{1+b} I^{a} f=I^{1} I^{a+b} f
$$

and this equivalence is an identity because both sides are continuous. Further, both sides are locally absolutely continuous. Theorem $\mathrm{D}$ now gives that $I^{b} I^{a} f$ exists. Using (7) and (20),

$$
I^{b} I^{a} f=I^{-1} I^{1+b} I^{a} f=I^{-1} I^{1+b+a} f=I^{b+a} f,
$$

the last of these steps following from Theorem 3 with $a$ and $b$ replaced by $1+b+a$ and -1 . This completes the proof of Theorem 4 .

COROLlary. If re $a=0, f$ is locally integrable and $I^{a} f$ exists, then $I^{-a} I^{a} f$ exists and is equivalent to $f$. (This proves again a part of Theorem E.)

For, using (6) and (7), $f=I^{-1} I^{1} f=I^{0} f=I^{a-a} f=I^{-a} I^{a} f$.

\section{The Second Index Law with No Derivatives of Imaginary Orders}

Most of our theorems from here on involve a slightly heavier restriction near the origin than local integrability. They are expressed in terms of the classes $Q_{q}$ defined in $\S 3$. 
(24) In all proofs we write, with or without warning, re $a=\alpha$, re $b=\beta$, re $c=\gamma$.

THEOREM 5. If $a+b+c=0$, re $a>0$, re $b<0$, re $c>0$ and $f \in Q_{-\gamma}$ where $\gamma=$ re $c$, then

$$
I^{c} x^{b} I^{a} f(x)={ }^{0} x^{-a} I^{-b} x^{-c} f(x),
$$

and both sides are in $Q_{-\gamma+\varepsilon}$ for each $\varepsilon>0$.

If also $f \in Q_{-\gamma-\eta}$ for some $\eta>0$, then both sides are in $Q_{-\gamma-\eta}$.

Subject to certain justifications,

$$
\begin{aligned}
I^{c} x^{b} I^{a} f(x) & =\int_{0}^{x} \frac{(x-s)^{c-1}}{\Gamma(c)} s^{b} d s \int_{0}^{s} \frac{(s-t)^{a-1}}{\Gamma(a)} f(t) d t \\
& =\int_{0}^{x} f(t) d t \int_{t}^{x} \frac{(x-s)^{c-1}}{\Gamma(c)} s^{b} \frac{(s-t)^{a-1}}{\Gamma(a)} d s \\
& =x^{-a} \int_{0}^{x} \frac{(x-t)^{-b-1}}{\Gamma(-b)} t^{-c} f(t) d t=x^{-a} I^{-b} x^{-c} f(x),
\end{aligned}
$$

the last line resulting from applying Lemma $G$. This proves (25) for all positive $x$ for which (26) is absolutely convergent. For the absolute convergence will both justify the inversion of order of integration at (26) and ensure the convergence of the integrals with which the chain of equations begins and ends.

To establish the absolute convergence of (26) for almost all positive $x$, we apply Lemma $G$ in a way similar to that above, but with $f(t)$ replaced by its modulus and $a, b, c$ by their respective real parts $\alpha, \beta, \gamma$; thus

$$
\int_{0}^{x}|f(t)| d t \int_{t}^{x} \frac{(x-s)^{\gamma-1}}{\Gamma(\gamma)} s^{\beta} \frac{(s-t)^{\alpha-1}}{\Gamma(\alpha)} d s=x^{-x} \int_{0}^{x} \frac{(x-t)^{-\beta-1}}{\Gamma(-\beta)} t^{-\gamma}|f(t)| d t
$$

This is finite for almost all $x$ by Theorem $A$, because the integral on the right is the $-\beta$ th integral of $t^{-\gamma}|f(t)|$, which is locally integrable by data; so it is also locally integrable. This provides, for almost all $x$, the justifications needed above.

By Theorem $\mathrm{K}$ with $q, c$ and $a$ replaced by $-\gamma,-b$ and $-c$, we see that $x^{-a} I^{-b} x^{-c} f(x)$ is in $Q_{-\gamma+\varepsilon}$ for each $\varepsilon>0$.

If $f \in Q_{-\gamma-\eta}$ for some $\eta>0, f \in Q_{-\gamma}$ so (25) still holds; and Theorem $\mathrm{K}$ with $q, c$ and $a$ replaced by $-\gamma-\eta,-b$ and $-c$ shows that $x^{-a} I^{-b} x^{-c} f(x)$ is in $Q_{-\gamma-\eta}$.

THEOREM 6. If $a+b+c=0$, re $a>0$, re $b<0$, re $c<0$ and $f \in Q_{0}$ then

$$
I^{c} x^{b} I^{a} f(x)={ }^{0} x^{-a} I^{-b} x^{-c} f(x),
$$

and both sides are in $Q_{0}$ (that is, are locally integrable).

If also $f \in Q_{-\eta}$ for some $\eta>0$ then both sides are in $Q_{-\eta}$. 
Writing $a^{\prime}, b^{\prime}, c^{\prime}$ and $\phi(x)$ in place of $-b,-a,-c$ and $x^{-c} f(x)$, and $\alpha^{\prime}, \beta^{\prime}, \gamma^{\prime}$ for re $a^{\prime}$, re $b^{\prime}$, re $c^{\prime}$,

$$
a^{\prime}+b^{\prime}+c^{\prime}=0, \alpha^{\prime}>0, \beta^{\prime}<0, \gamma^{\prime}>0 \text { and } \phi \in Q_{-\gamma^{\prime}} .
$$

So by Theorem 5

$$
I^{c^{\prime}} x^{b^{\prime}} I^{a^{\prime}} \phi(x)={ }^{0} \quad x^{-a^{\prime}} I^{-b^{\prime}} x^{-c^{\prime}} \phi(x) .
$$

By definition (6) this gives

$$
x^{b^{\prime}} I^{a^{\prime}} \phi(x)={ }^{0} I^{-c^{\prime}} x^{-a^{\prime}} I^{-b^{\prime}} x^{-c^{\prime}} \phi(x)
$$

provided the left side of (29) is locally integrable. Now the left side is $x^{-a} I^{-b} x^{-c} f(x)$; and Theorem $\mathrm{K}$ with $q, c$ and $a$ replaced by $0,-b$ and $-c$ shows that this function is in $Q_{0}$, that is, is locally integrable. So (29) is proved, and hence also (25) since it is the same equation. Further, one side of (29) has just been shown to be in $Q_{0}$, hence so are both.

If $f \in Q_{-\eta}$ for some $\eta>0$ then $f \in Q_{0}$, so that (25) still holds; and Theorem $\mathrm{K}$ with $q, c$ and $a$ replaced by $-\eta,-b$ and $-c$ shows that $x^{-a} I^{-b} x^{-c} f(x)$ is in $Q_{-\eta}$.

THEOREM 7. If $a+b+c=0$, re $a<0$, re $b<0$, re $c>0$, and $I^{a} f$ exists in $Q_{\beta}$ where $\beta=$ re $b$, then

$$
I^{c} x^{b} I^{a} f(x)={ }^{0} x^{-a} I^{-b} x^{-c} f(x),
$$

and both sides are in $Q_{-\gamma+\varepsilon}$ for each $\varepsilon>0$.

If also $I^{a} f \in Q_{\beta-\eta}$ for some $\eta>0$, then both sides are in $Q_{-\gamma-\eta}$.

Writing $a^{\prime}, b^{\prime}, c^{\prime}$ and $\phi(x)$ in place of $-a,-c,-b$ and $I^{a} f(x)$, and $\alpha^{\prime}, \beta^{\prime}, \gamma$ for re $a^{\prime}$, re $b^{\prime}$, re $c^{\prime}$ again,

$$
a^{\prime}+b^{\prime}+c^{\prime}=0, \alpha^{\prime}>0, \beta^{\prime}<0, \gamma^{\prime}>0 \text { and } \phi \in Q_{-\gamma^{\prime}} .
$$

So by Theorem 5

$$
I^{c^{\prime}} x^{b^{\prime}} I^{a^{\prime}} \phi(x)={ }^{0} x^{-a^{\prime}} I^{-b^{\prime}} x^{-c^{\prime}} \phi(x) .
$$

By (6) this is

$$
I^{-b} x^{-c} f(x)={ }^{0} x^{a} I^{c} x^{b} I^{a} f(x)
$$

which gives (25).

By Theorem $\mathrm{K}$ with $q, c, a$ and $f$ replaced by $\beta, c, b$ and $\phi, x^{a} I^{c} x^{b} \phi(x)$ is in $Q_{\beta+\varepsilon}$ for each $\varepsilon>0$; so $I^{c} x^{b} \phi(x)$ is in $Q_{\alpha+\beta+\varepsilon}$, that is, $I^{c} x^{b} I^{a} f(x)$ is in $Q_{-\gamma+\varepsilon}$.

If $I^{a} f \in Q_{\beta-\eta}$ for some $\eta>0$, Theorem $\mathrm{K}$ with $q, c, a$ and $f$ replaced by $\beta-\eta, c, b$ and $\phi$ shows that $x^{a} I^{c} x^{b} \phi(x)$ is in $Q_{\beta-\eta}$. Thus $I^{c} x^{b} I^{a} f(x)$ is in $Q_{a+\beta-\eta}=Q_{-\gamma-\eta}$. 
TheOREM 8. If $a+b+c=0$, re $a<0$, re $b>0$, re $c<0$, and either side exists in $Q_{\alpha}$ where $\alpha=$ re $a$, then

$$
I^{c} x^{b} I^{a} f(x)={ }^{0} x^{-a} I^{-b} x^{-c} f(x),
$$

and $f$ is in $Q_{x+\varepsilon}$ for each $\varepsilon>0$.

If also either side is in $Q_{\alpha-\eta}$ for some $\eta>0$, then $f$ is in $Q_{\alpha-\eta}$.

(i) Suppose the left side exists in $Q_{\alpha}$; denote it by $\phi(x)$. Its existence implies that of $I^{a} f$. Since $\gamma$ and $\alpha$ are negative, (6) gives

whence

$$
x^{b} I^{a} f(x)={ }^{0} I^{-c} \phi(x)
$$

and

$$
I^{a} f(x)={ }^{0} x^{-b} I^{-c} \phi(x),
$$

$$
f(x)={ }^{0} I^{-a} x^{-b} I^{-c} \phi(x) .
$$

Writing $a^{\prime}, b^{\prime}$ and $c^{\prime}$ in place of $-c,-b$ and $-a$, and $\alpha^{\prime}, \beta^{\prime}, \gamma^{\prime}$ for re $a^{\prime}$, re $b^{\prime}$, re $c^{\prime}$ continually,

$$
a^{\prime}+b^{\prime}+c^{\prime}=0, \alpha^{\prime}>0, \beta^{\prime}<0, \gamma^{\prime}>0 \text { and } \phi \in Q_{-\gamma^{\prime}} .
$$

So, by Theorem 5, (30) holds; this gives

$$
f(x)=x^{c} I^{b} x^{a} \phi(x), \text { and so } x^{-c} f(x)=I^{b} x^{a} \phi(x) .
$$

Since $x^{a} \phi(x)$ is locally integrable by definition of $Q_{\alpha}$, (6) gives

$$
I^{-b} x^{-c} f(x)={ }^{0} x^{a} \phi(x) .
$$

Thus the right side of (25) exists and is equivalent to $\phi$; this establishes (25).

By Theorem $\mathrm{K}$ with $q, c, a$ and $f$ replaced by $\alpha, b, a$ and $\phi,(31)$ gives that $f$ is in $Q_{\alpha+\varepsilon}$ for each $\varepsilon>0$.

If $\phi$ exists in $Q_{\alpha-\eta}$ for some $\eta>0$, then $\phi$ exists in $Q_{\alpha}$ and (25) holds as proved above. The application of Theorem $\mathrm{K}$ may now be made with $q$ replaced by $\alpha-\eta$ instead of by $\alpha$, and we obtain instead that $f \in Q_{\alpha-\eta}$.

(ii) Suppose the right side of (25) (and not necessarily the left) exists in $Q_{\alpha}$. Writing $a^{\prime}, b^{\prime}, c^{\prime}$ and $\psi(x)$ in place of $b, c, a$ and $I^{-b} x^{-c} f(x)$,

$$
a^{\prime}+b^{\prime}+c^{\prime}=0, \alpha^{\prime}>0, \beta^{\prime}<0, \gamma^{\prime}<0 \text { and } \psi \in Q_{0} .
$$

So by Theorem 6

$$
I^{c^{\prime}} x^{b^{\prime}} I^{a^{\prime}} \psi(x)={ }^{0} x^{-a^{\prime}} I^{-b^{\prime}} x^{-c^{\prime}} \psi(x)
$$

this gives

and so

$$
I^{a} x^{c} I^{b} I^{-b} x^{-c} f(x)={ }^{0} x^{-b} I^{-c} x^{-a} I^{-b} x^{-c} f(x),
$$




$$
x^{b} I^{a} f(x)={ }^{0} I^{-c} x^{-a} I^{-b} x^{-c} f(x) .
$$

Since $-\gamma>0$ this gives (25) by means of (6), noting that $x^{-a} I^{-b} x^{-c} f(x)$ is locally integrable because we are assuming it to be in $Q_{\alpha}$, a subclass of $Q_{0}$.

Thus (25) holds, and the left side is in $Q_{\alpha}$ since the right side is. Thus $f$ is in $Q_{\alpha+\varepsilon}$ for each $\varepsilon>0$, as proved under (i) using Theorem $\mathrm{K}$.

If the right side of (25) exists in $Q_{\alpha-\eta}$ for some $\eta>0$, it exists in $Q_{\alpha}$ and the above proof under (ii) establishes (25). So the left side exists in $Q_{\alpha-\eta}$ and $f$ is consequently in $Q_{\alpha-\eta}$ as in (i).

THEOREM 9. If $a+b+c=0$, re $a<0$, re $b>0$, re $c>0$ and $I^{a} f$ exists, then

$$
I^{c} x^{b} I^{a} f(x)={ }^{0} x^{-a} I^{-b} x^{-c} f(x),
$$

both sides are in $Q_{\alpha}$ where $\alpha=$ re $a$, and $f$ is in $Q_{\alpha+\varepsilon}$ for each $\varepsilon>0$.

If also $I^{a} f$ is in $Q_{-\eta}$ for some $\eta>0$, then both sides are in $Q_{\alpha-\eta}$ and so is $f$.

Writing $a^{\prime}, b^{\prime}, c^{\prime}$ and $\phi(x)$ in place of $-b,-a,-c$ and $x^{-c} f(x)$,

$$
a^{\prime}+b^{\prime}+c^{\prime}=0, \alpha^{\prime}<0, \beta^{\prime}>0, \gamma^{\prime}<0
$$

and $I^{-b^{\prime}} x^{-c^{\prime}} \phi(x)$ exists. This existence implies, by definition (6), that this function is locally integrable, and consequently $x^{-a^{\prime}} I^{-b^{\prime}} x^{-c^{\prime}} \phi(x)$ exists in $Q_{a^{\prime}}$. So, by Theorem 8 , (30) holds, that is

$$
I^{-c} x^{-a} I^{-b} x^{-c} f(x)={ }^{0} x^{b} I^{a} f(x) .
$$

Since $\gamma>0$ this gives (25). Theorem 8 also gives that $\phi$ is in $Q_{\alpha^{\prime}+\varepsilon}$ for each $\varepsilon>0$; thus $x^{-\beta+\varepsilon} x^{-c} f(x)$ is locally integrable, and so $f$ is in $Q_{\alpha+\varepsilon}$.

By Theorem $\mathrm{K}$ with $q, c, a$ and $f$ replaced by $0, c, b$ and $I^{a} f, x^{a} I^{c} x^{b} I^{a} f(x)$ is in $Q_{0}$, since $0<\mathrm{re} b$; consequently the left side of (25) is in $Q_{a}$. This completes the proof of the first paragraph of the statement.

For the second paragraph we suppose that $I^{a} f$ exists in $Q_{-\eta}$. The conclusions of the first paragraph still hold, but also $I^{-b^{\prime}} x^{-c^{\prime}} \phi(x)$ exists in $Q_{-\eta}$, so $x^{-a^{\prime}} I^{-b^{\prime}} x^{-c^{\prime}} \phi(x)$ exists in $Q_{\alpha^{\prime}-\eta}$. Then Theorem 8 gives that $\phi$ is in $Q_{-\beta-\eta}$, whence $x^{-\beta-\eta} x^{-c} f(x)$ is locally integrable and so $f$ is in $Q_{\alpha-\eta}$.

The above application of Theorem $\mathrm{K}$ may now be made with $q$ replaced by $-\eta$ instead of 0 ; it gives that $x^{a} I^{c} x^{b} I^{a} f(x)$ is in $Q_{-\eta}$, and so the left side of (25) is in $Q_{n-\eta}$. Since the two sides of (25) are equivalent as already mentioned, this completes the proof of Theorem 9.

Remark 1. The conclusions about $f$ are also deducible directly, without Theorem 8 , from the data that $I^{a} f$ is in $Q_{0}$ or in $Q_{-\eta}$, by applying Theorem $\mathrm{K}$ with $q, c, a$ and $f$ replaced by 0 or $-\eta,-a, 0$ and $I^{a} f$.

Remark 2. The conditions on $I^{a} f$ in Theorem 9 permit the application of Theorem 8 using the hypothesis about the right side. Instead we could impose 
conditions enabling Theorem 8 to be applied using the hypothesis about the left side. These conditions turn out to be more complicated; instead of $I^{a} f$ existing in $Q_{0}$ or in $Q_{-\eta}$ it is required that $I^{-c} x^{-a} I^{-b} x^{-c} f$ should exist in $Q_{-\beta}$ or in $Q_{-\beta-\eta}$. For simplicity the statement of Theorem 9 is not encumbered by these cases; and a similar policy of omitting such cases is followed in the rest of this paper.

THEOREM 10. If $a+b+c=0$, re $a>0$, re $b>0$, re $c<0, f$ is locally integrable and either side exists, then

$$
I^{c} x^{b} I^{a} f(x)={ }^{0} x^{-a} I^{-b} x^{-c} f(x) .
$$

If also either side is in $Q_{-\eta}$ for some $\eta>0$ then $f$ is in $Q_{-\eta}$.

First $I^{a} f$ exists by Theorem A. Writing $a^{\prime}, b^{\prime}, c^{\prime}$ and $\phi$ in place of $-a,-c$, $-b$ and $I^{a} f$,

$$
a^{\prime}+b^{\prime}+c^{\prime}=0, \alpha^{\prime}<0, \beta^{\prime}>0, \gamma^{\prime}<0 .
$$

Also $I^{-a} \phi=f$ by $(6)$, since $f$ is locally integrable.

(i) Suppose the left side of (25) exists. Then $I^{-b^{\prime}} x^{-c^{\prime}} \phi(x)$ is locally inte-

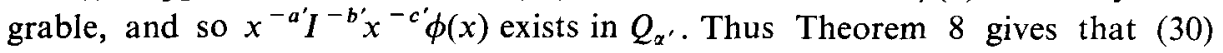
holds, that is,

$$
I^{-b} x^{-c} f(x)={ }^{0} x^{a} I^{c} x^{b} I^{a} f(x),
$$

which shows that (25) holds.

If the left side of (25) is in $Q_{-\eta}$ the previous paragraph still establishes (25); so the right side is in $Q_{-\eta}$ and $I^{-b} x^{-c} f(x)$ is in $Q_{-\alpha-\eta}$. By Theorem $\mathrm{K}$ with $q$, $c, a$ and $f(x)$ replaced by $-\alpha-\eta, b, 0$ and $I^{-b} x^{-c} f(x)$,

$$
x^{-b-c} f(x)={ }^{0} x^{-b} I^{b}\left(I^{-b} x^{-c} f\right)(x) \in Q_{-\alpha-\eta} ;
$$

thus $x^{-\eta} f(x)$ is locally integrable and $f \in Q_{-\eta}$.

(ii) Suppose the right side of (25) exists. Then $x^{a^{\prime}} I^{c^{\prime}} x^{b^{\prime}} I^{a^{\prime}} \phi(x)$ is locally integrable, and so $I^{c^{\prime}} x^{b^{\prime}} I^{a^{\prime}} \phi(x)$ exists in $Q_{\alpha^{\prime}}$. Again Theorem 8 gives that (30) holds, that is, (34) holds and hence so does (25).

If the right side of (25) is in $Q_{-\eta}$ the previous paragraph still establishes (25), and the paragraph before it shows that $f \in Q_{-\eta}$.

Remark 1. The applications of Theorem 8 in the above proof also give the information that $I^{a} f$ is in $Q_{-\alpha+\varepsilon}$ for each $\varepsilon>0$, or that $I^{a} f$ is in $Q_{-\alpha-\eta}$. These conclusions are omitted for simplicity, and also because they are directly deducible from the datum that $f$ is in $Q_{0}$ and the conclusion that $f$ is in $Q_{-\eta}$ by applications of Theorem $\mathrm{K}$ with $q, c$ and $a$ replaced by 0 or $-\eta, a$ and 0 .

Remark 2. It may seem that the hypotheses of Theorems 5 to 10 are dependent on the methods of proof, or on the order in which the theorems are 
proved, rather than that they are intrinsically connected with the theorems themselves. An indication to the contrary is available however. Theorem 7 can be deduced from Theorem 10, and the hypotheses which fit this method of proof are the same as before although our proof of Theorem 10 makes no use of Theorem 7 .

To prove Theorem 7 in this way, assume its hypotheses and write $a^{\prime}, b^{\prime}$, $c^{\prime}$ and $\phi(x)$ in place of $-b,-a,-c$ and $x^{-c} f(x)$. Then

$$
a^{\prime}+b^{\prime}+c^{\prime}=0, \alpha^{\prime}>0, \beta^{\prime}>0, \gamma^{\prime}<0 ;
$$

and $\phi \in Q_{0}$, because Theorem $\mathrm{K}$ with $q, c, a$ and $f$ replaced by $\beta,-a, 0$ and $I^{a} f$ gives that $x^{a} I^{-a}\left(I^{a} f\right)(x) \in Q_{\beta}$, and so

$$
\phi(x)=x^{-c} f(x)={ }^{0} x^{b} x^{a} I^{-a}\left(I^{a} f\right)(x)
$$

is locally integrable. The hypotheses of Theorem 7 further give that $I^{-b^{\prime}} x^{-c^{\prime}} \phi(x)$

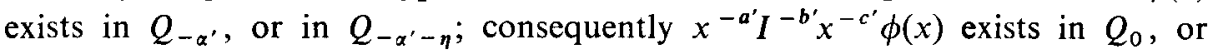
in $Q_{-\eta}$. Theorem 10 now gives that (30) holds; and from it (25) follows as shown at (33), the substitutions for $a^{\prime}, b^{\prime}, c^{\prime}$ and $\phi$ being the same there as here.

We have proved that $\phi \in Q_{0}$; and in the additional case Theorem 10 gives that $\phi \in Q_{-\eta}$. Thus $f$ is in $Q_{-\gamma}$, or in $Q_{-\gamma-\eta}$. Theorem $\mathrm{K}$ with $q, c$ and $a$ replaced by $-\gamma$ or $-\gamma-\eta,-b$ and $-c$ then shows that $x^{b+c} I^{-b} x^{-c} f(x)$ is in $Q_{-\gamma+\varepsilon}$ for each $\varepsilon>0$, or in $Q_{-\gamma-\eta}$; and this completes this second proof of Theorem 7 .

It is striking how all details of hypothesis and conclusion of Theorem 7 come naturally out of Theorem 10 in this proof, just as they come naturally out of Theorem 5 in the first proof. This suggests that these details have some objectivity.

\section{The Second Index Law with One Derivative of Imaginary Order}

In Theorems 5 and 6 we supposed $\alpha=$ re $a>0$ and $\beta=\operatorname{re} b<0$, and $\gamma=\operatorname{re} c$ took all values except 0 . Theorem 11 below will fill this gap by dealing with $\gamma=0$. We might expect some sort of continuity in hypothesis and conclusion as $\gamma$ passes through 0 , and this is in fact observed. Theorems 11 to 16 all have this character of providing a transition between some two of Theorems 5 to 10 .

THEOREM 11. If $a+b+c=0$, re $a>0$, re $b<0$, re $c=0$ and $f$ is in $Q_{-\eta}$ for some $\eta>0$, then

$$
I^{c} x^{b} I^{a} f(x)={ }^{0} x^{-a} I^{-b} x^{-c} f(x)
$$

and both sides are in $Q_{-\eta}$. [Transition between Theorems 5 and 6.]

First, the result is almost immediate if $c=0$. For then $a+b=0$ and both sides of (25) reduce to $x^{-a} I^{a} f(x)$. This exists for almost all $x>0$ by Theorem 
A, and it defines a function in $Q_{-\eta}$, by Theorem $\mathrm{K}$ with $q, c$ and $a$ replaced by $-\eta, a$ and 0 .

For the general case suppose that $c$ is any purely imaginary number except

0 . We show that the left and right sides of (25) have a common primitive.

The left side of (25) has, by (7), the primitive

$$
\begin{aligned}
I^{1+c} x^{b} I^{a} f(x) & =\int_{0}^{x} \frac{(x-s)^{c}}{\Gamma(1+c)} s^{b} d s \int_{0}^{s} \frac{(s-t)^{a-1}}{\Gamma(a)} f(t) d t \\
& =\int_{0}^{x} f(t) d t \int_{t}^{x} \frac{(x-s)^{c}}{\Gamma(c+1)} s^{b} \frac{(s-t)^{a-1}}{\Gamma(a)} d s
\end{aligned}
$$

certainly if the double integral (35) is absolutely convergent, which we show as follows. Since $\gamma=0$ and $\eta>0$,

$$
\begin{aligned}
& \int_{0}^{x}|f(t)| d t \int_{t}^{x}(x-s)^{\gamma} s^{\beta}(s-t)^{\alpha-1} d s \leqq \int_{0}^{x}|f(t)| d t \int_{t}^{x}(s-t)^{\alpha-1} s^{\beta}\left(\frac{s}{t}\right)^{\eta} d s \\
& \text { (36) } \quad=\int_{0}^{x} t^{-\eta}|f(t)| d t \int_{t}^{x}(s-t)^{\alpha-1} s^{\beta+\eta} d s \leqq M x^{\eta} \int_{0}^{x} t^{-\eta}|f(t)| d t
\end{aligned}
$$

where $M$ depends only on $\eta, \alpha$ and $\beta$, by Lemma $\mathrm{F}$ with $\lambda$ and $\mu$ replaced by $\alpha$ and $\beta+\eta$, so that $\lambda+\mu=\alpha+\beta+\eta=\eta>0$. The finiteness of (36) for all $x>0$ follows since $f \in Q_{-\eta}$; and so the inversion at (35) is justified. Further, the existence of $I^{1+c} x^{b} I^{a} f(x)$ for all $x>0$ is thus established by absolute convergence.

Applying Lemma $\mathrm{H}$ to (35),

$$
\begin{aligned}
I^{1+c} x^{b} I^{a} f(x) & =\int_{0}^{x} t^{-c} f(t) d t \int_{t}^{x} s^{-a} \frac{(s-t)^{-b-1}}{\Gamma(-b)} d s \\
& =\int_{0}^{x} s^{-a} d s \int_{0}^{s} \frac{(s-t)^{-b-1}}{\Gamma(-b)} t^{-c} f(t) d t \\
& =\int_{0}^{x} s^{-a} I^{-b} s^{-c} f(s) d s=I^{1} x^{-a} I^{-b} x^{-c} f(x),
\end{aligned}
$$

subject to the validity of (38), where the order of integration has been changed. This we justify by absolute convergence of (37), which is a consequence of the above inequalities leading to (36). For, since $\gamma=0, \alpha+\beta=0$ and $t^{-\gamma}=1$; so, as at (36),

$$
\begin{aligned}
\int_{0}^{x} t^{-\gamma}|f(t)| d t \int_{t}^{x} s^{-\alpha}(s-t)^{-\beta-1} d s & =\int_{0}^{x}|f(t)| d t \int_{t}^{x} s^{\beta}(s-t)^{\alpha-1} d s \\
& \leqq M x^{\eta} \int_{0}^{x} t^{-\eta}|f(t)| d t<\infty .
\end{aligned}
$$

This establishes (39) for all $x>0$, and it remains to show that we can differentiate both sides almost everywhere, so obtaining (25). 
The function $\psi(x)=x^{-a} I^{-b} x^{-c} f(x)$ is in $Q_{-\eta}$, by Theorem $\mathrm{K}$ with $q, c$ and $a$ replaced by $-\eta,-b$ and $-c$. In particular it is locally integrable, since $Q_{-\eta} \subset Q_{0}$. So, by (6), $\psi=I^{-1} I^{1} \psi$. Then (39) and (7) give

$$
\psi(x)={ }^{0} I^{-1} I^{1} \psi(x)=I^{-1} I^{1+c} x^{b} I^{a} f(x)=I^{c} x^{b} I^{a} f(x) .
$$

This proves the existence of the derivative of imaginary order involved, and completes the proof of Theorem 11 .

THEOREM 12. If $a+b+c=0$, re $a<0$, re $b=0$, re $c>0$ and $I^{a} f$ exists in $Q_{-\eta}$ for some $\eta>0$, then

$$
I^{c} x^{b} I^{a} f(x)={ }^{0} x^{-a} I^{-b} x^{-c} f(x),
$$

and $f$ and both sides are in $Q_{-\gamma-\eta}$, where $\gamma=$ re $c$. [Transition between Theorems 7 and 9.]

Writing $a^{\prime}, b^{\prime}, c^{\prime}$ and $\phi$ in place of $-a,-c,-b$ and $l^{a} f$,

$$
a^{\prime}+b^{\prime}+c^{\prime}=0, \alpha^{\prime}>0, \beta^{\prime}<0, \gamma^{\prime}=0 \text { and } \phi \in Q_{-\eta} .
$$

So by Theorem 11,

$$
I^{c^{\prime}} x^{b^{\prime}} I^{a^{\prime}} \phi(x)={ }^{0} x^{-a^{\prime}} I^{-b^{\prime}} x^{-c^{\prime}} \phi(x)
$$

and both sides are in $Q_{-\eta}$. The two sides are

$$
I^{-b} x^{-c} f(x)={ }^{0} x^{a} I^{c} x^{b} I^{a} f(x),
$$

which gives both (25) and that its two sides are in $Q_{\alpha-\eta}=Q_{-\gamma-\eta}$.

Since $x^{a} f(x)=x^{a} I^{-a} \phi(x)$ and $\phi \in Q_{-\eta}$, Theorem $\mathrm{K}$ with $q, c, a$ and $f$ replaced by $-\eta,-a, 0$ and $\phi$ shows that $x^{a} f(x) \in Q_{-\eta}$; and so $f \in Q_{\alpha-\eta}$ as required.

THEOREM 13. If $a+b+c=0$, re $a=0$, re $b>0$, re $c<0, f \in Q_{0}$ and either side exists in $Q_{-\eta}$ for some $\eta>0$, then

$$
I^{c} x^{b} I^{a} f(x)={ }^{0} x^{-a} I^{-b} x^{-c} f(x),
$$

and $f \in Q_{-\eta}$. [Transition between Theorems 8 and 10.]

(i) Suppose the left side exists in $Q_{-\eta}$. Writing $a^{\prime}, b^{\prime}, c^{\prime}$ and $\phi(x)$ in place of $c, a, b$ and $x^{b} I^{a} f(x)$,

$$
a^{\prime}+b^{\prime}+c^{\prime}=0, \quad \alpha^{\prime}<0, \beta^{\prime}=0, \gamma^{\prime}>0
$$

and $I^{a^{\prime}} \phi$ exists in $Q_{-\eta}$. So, by Theorem 12 , (30) holds and both sides are in $Q_{-\gamma^{\prime}-\eta}=Q_{-\beta-\eta}$. In fact the two sides are

$$
I^{b} x^{a} I^{c} x^{b} I^{a} f(x)={ }^{0} x^{-c} I^{-a} I^{a} f(x)={ }^{0} x^{-c} f(x) .
$$


The last step follows from Theorem 4 Cor., and is the place where we need the hypothesis that $f$ is locally integrable.

Since $x^{a}$ is bounded, $x^{a} I^{c} x^{b} I^{a} f(x) \in Q_{-\eta} \subset Q_{0}$; so, using (6), (40) gives

$$
x^{a} I^{c} x^{b} I^{a} f(x)={ }^{0} I^{-b} x^{-c} f(x),
$$

from which follows (25). Also $I^{-b} x^{-c} f(x) \in Q_{-\eta}$; applying Theorem $\mathrm{K}$ with $q, c, a$ and $f(x)$ replaced by $-\eta, b, 0$ and $I^{-b} x^{-c} f(x)$, we obtain the conclusion that $x^{-b-c} f(x) \in Q_{-\eta}$, and so $f \in Q_{-\eta}$.

(ii) Suppose the right side exists in $Q_{-\eta}$. Writing $a^{\prime}, b^{\prime}, c^{\prime}$ and $\psi(x)$ in place of $-b,-a,-c$ and $x^{-c} f(x)$,

$$
a^{\prime}+b^{\prime}+c^{\prime}=0, \alpha^{\prime}<0, \beta^{\prime}=0, \gamma^{\prime}>0
$$

and $I^{a^{\prime}} \psi$ exists in $Q_{-\eta}$. So, by Theorem 12, (32) holds and both sides are in $Q_{-\gamma^{\prime}-\eta}=Q_{\gamma-\eta}$. In fact the two sides are

$$
I^{-c} x^{-a} I^{-b} x^{-c} f(x)={ }^{0} x^{b} I^{a} f(x) .
$$

Since the right side of (25) is in $Q_{-\eta}$ it is locally integrable; so, using (6), (41) gives (25).

We prove that $f \in Q_{-\eta}$ exactly as in the last sentence of (i).

THEOREM 14. If $a+b+c=0$, re $a=0$, re $b<0$, rec $>0, f$ is in $Q_{-y}$ and $I^{a} f$ exists in $Q_{-\gamma}$, where $\gamma=\operatorname{re} c$, then

$$
I^{c} x^{b} I^{a} f(x)={ }^{0} x^{-a} I^{-b} x^{-c} f(x),
$$

and both sides are in $Q_{-\gamma+\varepsilon}$ for each $\varepsilon>0$.

If also either $f$ or $I^{a} f$ is in $Q_{-\gamma-\eta}$ for some $\eta>0$, then both sides are in $Q_{-\gamma-\eta}$. [Transition between Theorems 5 and 7. Observe that $Q_{\beta}=Q_{-\gamma}$ and that $\left.Q_{\beta-\eta}=Q_{-\gamma-\eta} \cdot\right]$

Since $Q_{-\gamma} \subset Q_{0}, f$ is locally integrable. By Theorem 3 with $a$ and $b$ replaced by 1 and $a-1$,

$$
I^{a-1} I^{1} f=I^{a} f .
$$

Since also $I^{a} f \in Q_{-\gamma}=Q_{\beta}, I^{a-1} I^{1} f$ exists in $Q_{\beta}$. Using Theorem 7 with $a, c$ and $f$ replaced by $a-1, c+1$ and $I^{1} f$,

$$
I^{c+1} x^{b} I^{a} f(x)=I^{c+1} x^{b} I^{a-1} I^{1} f(x)={ }^{0} x^{1-a} I^{-b} x^{-c-1} I^{1} f(x) .
$$

Since $f \in Q_{-\gamma}, x^{-c} f(x)$ is locally integrable. Theorem $\mathrm{K}$, with $q, c, a$ and $f(x)$ replaced by $0,1, c$ and $x^{-c} f(x)$, thus gives that $x^{-c-1} I^{1} f(x)$ is also locally integrable. Then Theorem 1, with $a, b$ and $f(x)$ replaced by $c, a$ and $x^{-c-1} I^{1} f(x)$, gives that

$$
I^{a} I^{c} x^{-c-1} I^{1} f(x)={ }^{0} I^{c+a} x^{-c-1} I^{1} f(x)=I^{-b} x^{-c-1} I^{1} f(x) .
$$


Since $f \in Q_{-\gamma}$, Theorem 5 with $a$ and $b$ replaced by 1 and $-c-1$ gives that

$$
I^{c} x^{-c-1} I^{1} f(x)={ }^{0} x^{-1} I^{c+1} x^{-c} f(x) .
$$

Equations (42), (43), (44) now give

$$
I^{c+1} x^{b} I^{a} f(x)={ }^{0} x^{1-a} I^{a} x^{-1} I^{c+1} x^{-c} f(x) .
$$

Theorem $\mathrm{K}$ with $q, c$ and $a$ replaced by $-\gamma,-b$ and $-c$ shows that $x^{-a} I^{-b} x^{-c} f(x)$ is in $Q_{-\gamma+\varepsilon}$ for each $\varepsilon>0$, which is one of the stated conclusions. Since $\left|x^{-a}\right|=1$ it follows that $I^{-b} x^{-c} f(x)$ is also in $Q_{-\gamma+\varepsilon}$ for each $\varepsilon>0$; in particular it is locally integrable.

We have already seen that $x^{-c} f(x)$ is locally integrable. Theorem 1 , with $a, b$ and $f(x)$ replaced by $c+1, a-1$ and $x^{-c} f(x)$, gives that

$$
I^{a-1} I^{c+1} x^{-c} f(x)={ }^{0} I^{c+a} x^{-c} f(x)=I^{-b} x^{-c} f(x) .
$$

This with the preceding paragraph shows that, taking $\varepsilon=\frac{1}{2} \gamma=\eta>0$, the left side of (46) is in $Q_{-\eta}$; so Theorem 12 can be applied with $a, b, c$ and $f(x)$ replaced by $a-1,-a, 1$ and $I^{c+1} x^{-c} f(x)$. This gives

$$
I^{1} x^{-a} I^{a-1} I^{c+1} x^{-c} f(x)={ }^{0} x^{1-a} I^{a} x^{-1} I^{c+1} x^{-c} f(x) .
$$

Equations (45), (46), (47) now give

$$
I^{c+1} x^{b} I^{a} f(x)={ }^{0} I^{1} x^{-a} I^{-b} x^{-c} f(x) .
$$

Also $x^{-a} I^{-b} x^{-c} f(x)$ was shown above to be locally integrable; so (6) gives

$$
I^{-1} I^{c+1} x^{b} I^{a} f(x)={ }^{0} x^{-a} I^{-b} x^{-c} f(x) .
$$

Now $x^{b} I^{a} f(x)$ is locally integrable because $I^{a} f$ is in $Q_{\beta}$. Therefore Theorem 1 , with $a, b$ and $f(x)$ replaced by $c+1,-1$ and $x^{b} I^{a} f(x)$, gives that the left side of (48) is equivalent to that of (25). So (25) is established, and this completes the proof of the main paragraph of Theorem 14 .

If $f$ is in $Q_{-\gamma-\eta}$ for some $\eta>0$, in addition to the main hypotheses, Theorem $\mathrm{K}$ with $q, c$ and $a$ replaced by $-\gamma-\eta,-b$ and $-c$ shows that the right side of (25) is in $Q_{-\gamma-\eta}$; hence so are both sides.

If instead $I^{a} f$ is in $Q_{-\gamma-\eta}$ in addition to the main hypotheses, Theorem $\mathrm{K}$ with $q, a$ and $f$ replaced by $-\gamma-\eta, b$ and $I^{a} f$ shows that $x^{a} I^{c} x^{b} I^{a} f(x)$ is in $Q_{-\gamma-\eta}$. Since $\left|x^{a}\right|=1$, the left side of $(25)$ is also in $Q_{-\gamma-\eta}$; and hence so are both sides. This completes the proof of Theorem 14.

THEOREM 15. If $a+b+c=0$, re $a>0$, re $b=0$, re $c<0, f$ is locally integrable, and either:

(i) the right side exists and is locally integrable; or

(ii) $I^{-b} f$ exists in $Q_{-\eta}$, for some $\eta>0$; or

(iii) the left side exists in $Q_{-\eta}$, for some $\eta>0$, and $I^{-b} f$ exists; then 


$$
I^{c} x^{b} I^{a} f(x)={ }^{0} x^{-a} I^{-b} x^{-c} f(x) \text {. }
$$

[Transition between Theorems 6 and 10.] [See also Theorem 17.]

Case (i). Since $-\gamma>0, x^{-c}$ is locally bounded; so $x^{-c} f(x)$ is locally integrable. Also $\phi(x)=I^{-b} x^{-c} f(x)$ exists by data. So, by Theorem E, $I^{b} \phi$ exists and

$$
I^{b} \phi(x)={ }^{0} x^{-c} f(x) .
$$

Writing $a^{\prime}, b^{\prime}, c^{\prime}$ and $\phi(x)$ in place of $b, c, a$ and $I^{-b} x^{-c} f(x)$,

$$
a^{\prime}+b^{\prime}+c^{\prime}=0, \alpha^{\prime}=0, \beta^{\prime}<0, \gamma^{\prime}>0 .
$$

Now $x^{-a} \phi(x)$ is locally integrable, being the right side of (25), so $\phi \in Q_{-\alpha}=Q_{-\gamma}$. Also $x^{-c} f(x) \in Q_{\gamma}$ since $f$ is locally integrable; so (49) shows that $I^{a^{\prime}} \phi \in Q_{\gamma}=Q_{-\alpha}$ $=Q_{-\gamma}$. Theorem 14 now establishes (30), so that

and so

$$
I^{a} x^{c} I^{b} \phi(x)={ }^{0} x^{-b} I^{-c} x^{-a} \phi(x),
$$

$$
x^{b} I^{a} f(x)={ }^{0} I^{-c} x^{-a} \phi(x) .
$$

Since $x^{-a} \phi(x)$ is locally integrable, this by (6) gives

which is (25).

$$
I^{c} x^{b} I^{a} f(x)={ }^{0} x^{-a} \phi(x),
$$

Case (ii). Now $x^{-c} I^{-b} f(x)$ exists in $Q_{\gamma-\eta}$, and $f$ is locally integrable. Theorem $M$ with $q, a$ and $g(x)$ replaced by $\gamma-\eta, c$ and $x^{-c} f(x)$ gives that $I^{-b} x^{-c} f(x)$ exists in $Q_{\gamma-\eta}$. So the right side of (25) exists in $Q_{-\eta}$, hence in $Q_{0}$, and Case (i) applies.

Case (iii). Since $x^{-b}$ is bounded, $x^{-b} I^{c} x^{b} I^{a} f(x)$ exists in $Q_{-\eta}$. Theorem L with $q, a, b$ and $g$ replaced by $-\eta, b,-c$ and $I^{a} f$ shows that $I^{c} I^{a} f$ exists in $Q_{-\eta}$. By data $I^{c+a} f$ exists, and $\operatorname{re}(c+a)=-\mathrm{re} b=0$, so Theorem 3 with $b$ replaced by $c$ gives that $I^{c+a} f=I^{c} I^{a} f \in Q_{-\eta}$. Thus $I^{-b} f \in Q_{-\eta}$ and Case (ii) applies.

THEOREM 16. If $a+b+c=0$, re $a<0$, re $b>0$, re $c=0$ and either:

(i) the left side exists in $Q_{-\beta}$, where $\beta=\mathrm{re} b$; or

(ii) the right side exists, and $I^{a} f$ exists in $Q_{-\eta}$ for some $\eta>0$; or

(iii) the right side exists in $Q_{-\beta-\eta}$ for some $\eta>0$, and $I^{a} f$ exists; then

$$
I^{c} x^{b} I^{a} f(x)={ }^{0} x^{-a} I^{-b} x^{-c} f(x) .
$$

In case (i) $f$ is in $Q_{-\beta+\varepsilon}$ for each $\varepsilon>0$, while in cases (ii) and (iii) $f$ is in $Q_{-\beta-\eta}$. [Transition between Theorems 8 and 9.]

Case (i). Since the left side exists, $I^{a} f$ exists; thus $I^{a} f$ is locally integrable by (6), and $x^{b} I^{a} f(x) \in Q_{-\beta}$. Writing $a^{\prime}, b^{\prime}, c^{\prime}$ and $\phi(x)$ for $c, a, b$ and $x^{b} I^{a} f(x)$,

$$
a^{\prime}+b^{\prime}+c^{\prime}=0, \alpha^{\prime}=0, \beta^{\prime}<0, \gamma^{\prime}>0,
$$


$\phi \in Q_{-\gamma^{\prime}}$ and $I^{a^{\prime}} \phi=I^{c} x^{b} I^{a} f(x)$ exists in $Q_{-\gamma^{\prime}}$. Theorem 14 now establishes (30), so that, using (6) to simplify the right side,

$$
I^{b} x^{a} I^{c} x^{b} I^{a} f(x)={ }^{0} x^{-c} f(x) .
$$

By hypothesis $x^{a} I^{c} x^{b} I^{a} f(x)$ is locally integrable, since $\left|x^{a}\right|=x^{\alpha}=x^{-\beta}$. So using (6) we obtain

$$
x^{a} I^{c} x^{b} I^{a} f(x)={ }^{0} I^{-b} x^{-c} f(x),
$$

and from this (25) follows.

As remarked above, $I^{a} f \in Q_{0}$. By Theorem $\mathrm{K}$ with $q, c, a$ and $f$ replaced by $0,-a, 0$ and $I^{a} f, x^{a} I^{-a} I^{a} f(x)$ is in $Q_{\varepsilon}$ for each $\varepsilon>0$. But $I^{-a} I^{a} f=f$ by (6), and so $f$ is in $Q_{\alpha+\varepsilon}=Q_{-\beta+\varepsilon}$.

Case (ii). Since $I^{-b} x^{-c} f(x)$ exists it is, by (6), a locally integrable function $\chi$ such that $x^{-c} f(x)=I^{b} \chi(x)$. And $I^{b} \chi$ is locally integrable by Theorem $\mathrm{A}$, so that $x^{-c} f(x)$ is locally integrable. Thus both $x^{-c} f(x)$ and $I^{-b} x^{-c} f(x)$ are locally integrable.

Theorem $\mathrm{L}$ with $q, a, b$ and $g$ replaced by $-\eta,-c,-a$ and $f$ shows that $x^{c} I^{a} x^{-c} f(x)$ exists in $Q_{-\eta}$, because $I^{a} f$ exists in $Q_{-\eta}$ by hypothesis. Also $\left|x^{c}\right|=1$ and $a=-c-b$, so $I^{-c-b} x^{-c} f(x)$ exists in $Q_{-\eta}$.

Theorem 4 with $a, b$ and $f(x)$ replaced by $-b,-c$ and $x^{-c} f(x)$ now gives that

$$
I^{-c} I^{-b} x^{-c} f(x)={ }^{0} I^{-c-b} x^{-c} f(x) ;
$$

so the left side also of this equation exists in $Q_{-\eta}$.

Writing $a^{\prime}, b^{\prime}, c^{\prime}$ and $\psi(x)$ in place of $b, c, a$ and $I^{-b} x^{-c} f(x)$,

$$
a^{\prime}+b^{\prime}+c^{\prime}=0, \quad \alpha^{\prime}>0, \beta^{\prime}=0, \gamma^{\prime}<0,
$$

$\psi$ is locally integrable, and $I^{-b^{\prime}} \psi$ exists in $Q_{-\eta}$ by (50). So Theorem 15 (ii) shows that (32) holds; this is, using (6) to simplify the left side,

$$
I^{a} f(x)={ }^{0} x^{-b} I^{-c} x^{-a} \psi(x) .
$$

Since $\psi$ is locally integrable $x^{-a} \psi(x) \in Q_{\alpha} \subset Q_{0}$. Also $I^{-c} x^{-a} \psi(x)$ exists by (51). Theorem 4 Cor. then gives that $I^{c} I^{-c} x^{-a} \psi(x)$ exists and is equivalent to $x^{-a} \psi(x)$. That is, using (51),

$$
x^{-a} I^{-b} x^{-c} f(x)=x^{-a} \psi(x)={ }^{0} I^{c} x^{b} I^{a} f(x),
$$

which proves (25).

By Theorem $\mathrm{K}$ with $q, c, a$ and $f$ replaced by $-\eta,-a, 0$ and $I^{a} f, x^{a} I^{-a} I^{a} f$ is in $Q_{-\eta}$. But $I^{-a} I^{a} f=f$ by (6), so $f$ is in $Q_{\alpha-\eta}=Q_{-\beta-\eta}$.

Case (iii). Since $x^{-a} I^{-b} x^{-c} f(x)$ exists in $Q_{a-\eta}, x^{c} I^{-b} x^{-c} f(x)$ exists in $Q_{-\gamma-\eta}=Q_{-\eta}$. By Theorem $\mathrm{L}$ with $q, a$ and $g$ replaced by $-\eta,-c$ and $f, I^{-b} f$ exists in $Q_{-\eta}$; that is, $I^{c+a} f$ exists in $Q_{-\eta}$. 
The first paragraph of (ii) is still effective in showing that $x^{-c} f(x)$ is locally integrable. Since $\left|x^{-c}\right|=1, f$ itself is locally integrable. Since $I^{a} f$ and $I^{c+a} f$ exist, Theorem 4 shows that

$$
I^{c} I^{a} f=I^{c+a} f .
$$

It follows that $I^{c} I^{a} f$ exists in $Q_{-\eta}$; and hence that $x^{b} I^{c} I^{a} f$ exists in $Q_{-\beta-\eta}$. Also $I^{a} f$ is locally integrable by (6). Further $I^{-b} f$ exists in $Q_{-\eta}$.

Theorem $\mathrm{M}$ with $q, a, b$ and $g(x)$ replaced by $-\beta-\eta,-b,-c$ and $x^{b} I^{a} f(x)$ is now applicable, and gives that $I^{c} x^{b} I^{a} f(x)$ exists in $Q_{-\beta-\eta}$. In particular the left side of (25) exists in $Q_{-\beta}$, and so (i) shows that (25) holds.

Theorem $\mathrm{K}$ with $q, c, a$ and $f$ replaced by $-\eta, b, 0$ and $I^{-b} f$ shows that $x^{-b} I^{b} I^{-b} f(x)$ is in $Q_{-\eta}$. Using (6) we obtain that $f$ is in $Q_{-\beta-\eta}$. This completes the proof of Theorem 16 .

THEOREM 17. If $a+b+c=0$, re $a>0$, re $b=0$, re $c<0, f$ is in $Q_{-\eta}$ for some $\eta>0$ and the left side exists, then

$$
I^{c} x^{b} I^{a} f(x)={ }^{0} x^{-a} I^{-b} x^{-c} f(x) .
$$

[An extra case of Theorem 15, proved using Theorem 16.]

Noting that $I^{a}$ exists, by data or by Theorem A, consider the function $\psi(x)=x^{b} I^{a} f(x)$. Since $f$ is locally integrable, (6) gives

$$
f(x)={ }^{0} I^{-a} x^{-b} \psi(x) .
$$

Since $f$ is in $Q_{-\eta}, x^{-c} I^{-a} x^{-b} \psi(x)$ is in $Q_{\gamma-\eta}=Q_{-\alpha-\eta}$.

Writing $a^{\prime}, b^{\prime}, c^{\prime}$ in place of $c, a, b$,

$$
a^{\prime}+b^{\prime}+c^{\prime}=0, \alpha^{\prime}<0, \beta^{\prime}>0, \gamma^{\prime}=0 .
$$

Then $x^{-a^{\prime}} I^{-b^{\prime}} x^{-c^{\prime}} \psi(x)$ is in $Q_{-\beta^{\prime}-\eta}$, and $I^{a^{\prime}} \psi(x)=I^{c} x^{b} I^{a} f(x)$ exists. So Theorem 16 (iii) shows that (32) holds; that is,

$$
I^{b} x^{a} I^{c} \psi(x)={ }^{0} x^{-c} f(x),
$$

using (52) to simplify the right side.

Now $I^{c} \psi$ exists, being the left side of (25). By (6) it is locally integrable. And since $x^{a}$ is locally bounded, $x^{a} I^{c} \psi(x)$ is also locally integrable. Since the left side of (53) exists, Theorem $E$ now establishes the equation

$$
x^{a} I^{c} \psi(x)={ }^{0} I^{-b} x^{-c} f(x),
$$

which by a minor transformation becomes (25). 


\section{The Second Index Law with More than One Derivative of Imaginary Order}

Theorems 5 to 16 establish the second index law, under suitable conditions, for twelve different combinations of sign of the real parts of the orders of integration $a, b, c$. Theorem 17 repeats the sign combination of Theorem 15 , but with different conditions. The only remaining sign combination consistent with $a+b+c=0$ is that in which $a, b, c$ are all imaginary, and this combination is considered in the remaining theorem.

THEOREM 18. If $a+b+c=0$, re $a=0$, re $b=0$, re $c=0, \eta>0, f$ is in $Q_{-\eta}, I^{a} f$ exists in $Q_{-\eta}$, and either side exists in $Q_{-\eta}$, then

$$
I^{c} x^{b} I^{a} f(x)={ }^{0} x^{-a} I^{-b} x^{-c} f(x) .
$$

(i) Suppose that the right side of (25) exists in $Q_{-\eta}$. Since $Q_{-\eta} \subset Q_{0}, f$ is locally integrable. By Theorem 3 with $a$ and $b$ replaced by 1 and $a-1$, respectively $I^{a-1} I^{1} f=I^{a} f$. Thus $I^{a-1} I^{1} f$ exists in $Q_{-\eta}$; and so by Theorem 12 with $a, c$ and $f$ replaced by $a-1, c+1$ and $I^{1} f$,

$$
I^{c+1} x^{b} I^{a} f(x)={ }^{0} I^{c+1} x^{b} I^{a-1} I^{1} f(x)={ }^{0} x^{1-a} I^{-b} x^{-c-1} I^{1} f(x) .
$$

By Theorem 11 with $a$ and $b$ replaced by 1 and $-c-1$,

$$
I^{c} x^{-c-1} I^{1} f(x)={ }^{0} x^{-1} I^{c+1} x^{-c} f(x)
$$

Further, Theorem $\mathrm{K}$ with $q, c$ and $a$ replaced by $-\eta, 1$ and 0 shows that $x^{-1} I^{1} f(x)$ is in $Q_{-\eta}$; from which it follows, since $\left|x^{-c}\right|=1$ and $Q_{-\eta} \subset Q_{0}$, that $x^{-c-1} I^{1} f(x)$ is locally integrable. This, with the existence of $I^{c} x^{-c-1} I^{1} f(x)$ and $I^{a+c} x^{-c-1} I^{1} f(x)$ established in (55) and (54), shows that

$$
I^{a} I^{c} x^{-c-1} I^{1} f(x)={ }^{0} I^{a+c} x^{-c-1} I^{1} f(x)=I^{-b} x^{-c-1} I^{1} f(x),
$$

by Theorem 4 with $a, b$ and $f(x)$ replaced by $c, a$ and $x^{-c-1} I^{1} f(x)$. Equations (54), (55) and (56) now give

$$
I^{c+1} x^{b} I^{a} f(x)={ }^{0} x^{1-a} I^{a} x^{-1} I^{c+1} x^{-c} f(x) .
$$

Now $I^{-b} x^{-c} f(x)$ exists, by the assumed existence of the right side of (25), so that $I^{a+c} x^{-c} f(x)$ exists. By Theorem 3 with $a, b$ and $f(x)$ replaced by $c+1$, $a-1$ and $x^{-c} f(x)$,

$$
I^{-b} x^{-c} f(x)=I^{a+c} x^{-c} f(x)={ }^{0} I^{a-1} I^{c+1} x^{-c} f(x) .
$$

In particular this, or Theorem A, establishes the existence of $I^{c+1} x^{-c} f(x)$.

Again $I^{-b} x^{-c} f(x)$ is in $Q_{-\eta}$, since the right side of $(25)$ is. So the right side of (58) is also in $Q_{-\eta}$. By Theorem 12 with $a, b, c$ and $f(x)$ replaced by $a-1$, $-a, 1$ and $I^{c+1} x^{-c} f(x)$, 


$$
I^{1} x^{-a} I^{a-1} I^{c+1} x^{-c} f(x)={ }^{0}: x^{1-a} I^{a} x^{-1} I^{c+1} x^{-c} f(x)
$$

Equations (57), (58) and (59) now give

$$
I^{c+1} x^{b} I^{a} f(x)={ }^{0} I^{1} x^{-a} I^{a+c} x^{-c} f(x) .
$$

Also $x^{-a} I^{a+c} x^{-c} f(x)$ is locally integrable, being the right side of (25); so (6) gives

$$
I^{-1} I^{1+c} x^{b} I^{a} f(x)={ }^{0} x^{-a} I^{-b} x^{-c} f(x),
$$

establishing the existence of $I^{c} x^{b} I^{a} f(x)$ in accordance with (7), and proving (25).

This proof runs closely parallel to that of Theorem 14; in fact, equations (54) to (59) are the same as equations (42) to (47), with a minor difference in order. But the reasons leading to the equations are mostly different, or rest on different theorems.

(ii) Suppose now, instead, that the left side of (25) exists in $Q_{-\eta}$. Writing $a^{\prime}, b^{\prime}, c^{\prime}$ and $\phi(x)$ in place of $c, a, b$ and $x^{b} I^{a} f(x)$,

$$
a^{\prime}+b^{\prime}+c^{\prime}=0, \quad \alpha^{\prime}=0, \beta^{\prime}=0, \gamma^{\prime}=0, \eta>0,
$$

$\phi$ is in $Q_{-\eta}$ and $I^{a^{\prime}} \phi$ exists in $Q_{-\eta}$. Theorem 4 Cor. gives that

$$
x^{-a^{\prime}} I^{-b^{\prime}} x^{-c^{\prime}} \phi(x)=x^{-c} I^{-a} I^{a} f(x)={ }^{0} x^{-c} f(x) ;
$$

so that the right side of (30) also exists in $Q_{-\eta}$. Thus (i) above applies to show that (30) holds; that is

$$
I^{b} x^{a} I^{c} x^{b} I^{a} f(x)={ }^{0} x^{-c} f(x) .
$$

Since $I^{c} x^{b} I^{a} f(x)$ exists in $Q_{-\eta}$ it is locally integrable; and since $\left|x^{a}\right|=1$ $x^{a} I^{c} x^{b} I^{a} f(x)$ is locally integrable. So $(60)$ gives, by Theorem 4 Cor.,

$$
x^{a} I^{c} x^{b} I^{a} f(x)={ }^{0} I^{-b} x^{-c} f(x),
$$

and this establishes (25), as required.

Acknowledgment. I am indebted to Prof. Erdélyi for the simple proof given of Lemma G. This led me to simplifications in the proofs I previously had of Theorems 5, 6 and 11 .

Erdélyi has also proved both index laws for fractional integrals of certain classes of generalized function. His approach has the advantage that the separate treatment of numerous cases is unnecessary. On the other hand, his conclusions fall short in that, under my hypotheses, his treatment does not show that the generalized functions on each side of each index law are ordinary functions in the specified $Q$-classes, equal almost everywhere. 


\section{References}

[1] D. V. Widder, 'The Stieltjes Transform', Trans. American Math. Soc. 43 (1938), 7-60.

[2] E. R. Love and L. C. Young, 'On fractional integration by parts', Proc. London Math. Soc. (2) 44 (1938), 1-35.

[3] E. Hille, 'Notes on linear transformations: II Analyticity of semigroups', Annals of Math. (2) 40 (1939), 1-47.

[4] H. Kober, 'On fractional integrals and derivative', Quart. J. Math. (Oxford) XI (1940) 193-211.

[5] H. Kober, 'On a theorem of Schur and on fractional integrals of purely imaginary order,' Trans. American Math. Soc. 50 (1941), 160-174.

[6] M. Riesz, 'L'intégrale de Riemann-Liouville et le problème de Cauchy', Acta Math. 81 (1949), 1-223.

[7] A. Erdélyi et al., Higher Transcendental Functions, vol. 1 (McGraw Hill, 1953).

[8] I. M. Gelfand and G. E. Shilov, Generalized Functions, vol. 1 (Academic Press, 1963).

[9] T. P. Higgins, The Rodrigues Operator Transform, (Boeing Scientific Research Laboratories, Math. Note No. 437 (1965) 1-125.)

[10] A. Erdélyi, 'Some integral equations involving finite parts of divergent integrals', Glasgow Math. J. 8 (1967), 50-54.

[11] E. R. Love, 'Some integral equations involving hypergeometric functions,' Proc. Edinburgh Math. Soc. (II) 15 (1967), 169-198.

[12] E. R. Love, 'Fractional derivatives of imaginary order', J. London Math. Soc. (2) 3 (1971) 241-259.

Department of Mathematics

The University of Melbourne

Victoria, 3052

Australia 